\title{
EDITORIAL \\ When commonsense does not make sense
}

International Journal of Obesity (2012) 36, 1332-1333; doi:10.1038/ ijo.2012.100

'Will power is not a prerogative of thin people' and 'providing calorie information as a policy, may not be the sharpest sword to fight the obesity epidemic' appear to be the paraphrased conclusions of two articles published in this issue of the journal. ${ }^{1,2}$ Loeber et al. ${ }^{1}$ questioned the notion that links obesity to addictive behavior. It seems intuitive to link obesity to food addiction, and easy to stereotype obese individuals as preoccupied with food. Using obese and normal-weight healthy subjects and food or non-food related (neutral) pictures as stimuli, Loeber et al. ${ }^{1}$ demonstrated that indeed, food related stimuli are more pleasant than neutral stimuli, but that was equally true for both the groups. Unlike the previous studies that have reported a predilection of alcoholics or smokers to alcohol or smoking related stimuli, respectively, attention to food stimuli was similar in the obese and non-obese subjects. There was no significant difference between the two groups, for hunger scale, restraint or impulsivity. Overall, the results of this study combined with earlier brain-imaging reports, which show high-calorie foods light up reward centers in the brain, propose that as food is essential for survival, it is connected with neural circuitry of reward and pleasure. However, thoughts about food may not be processed differently in obese vs non-obese individuals.

The article by Girz et al., ${ }^{2}$ addresses the push to legislate calorie information for restaurant menus in the hope of fighting obesity. The policy that calls for listing of calories and suggested daily caloric intake on menus is based on obvious-sounding assumptions that such nutrition information will (1) change food intake at that meal, and that (2) the change will be only in one direction-lower. Considering the far-reaching influence of any legal directive, one would expect to see convincing, evidencedbased data and rationale before mandating changes. Else, well-intentioned policies could also have unintended adverse consequences. A recent systematic review identified surprisingly few studies about menu labeling and food intake. ${ }^{3}$ This review ${ }^{3}$ concluded that '...calorie menu labeling does not have the intended effect of decreasing calorie ordering and consumption from quick-service restaurants'. Girz et $a l^{2}{ }^{2}$ now add information about food selection and intake by restrained and unrestrained eaters, when calorie information is provided about salad and pasta, which are traditionally perceived to be healthful vs high-calorie foods. Overall, the study shows that calorie information may have varying effects on behavior related to the selection of food and its consumption. Unrestrained and restrained eaters differ in their food selection responses and no clear benefit seems to emerge from this information. Based on the available reports, the current vote does not favor usefulness of restaurant menu labeling. Swartz et al. ${ }^{3}$ cautioned that, 'we must proceed with caution in widespread implementation of an unproven policy with social and monetary costs, especially since the effort may detract attention from other effective strategies to combat overweight and obesity or have inadvertent effects'.

These two studies ${ }^{1,2}$ do not settle respective issues and additional and larger studies are needed. Yet, they are important because they challenge conventional thinking and commonsense.
One study questions the validity of the obese stereotype, and the other suggests that well-intentioned, commonsense solutions may be too simplistic to counter the obesity epidemic. Such studies call for an assessment of the field of obesity research. Perhaps, some other conventional approaches, although well-intentioned, intuitive and deeply entrenched, provide nothing more than a false sense of accomplishment, and thus impede the need to develop better strategies.

Despite extensive research and increasing social awareness, the prevalence of obesity has increased globally with no respite in sight. A view point is offered here to initiate discussions about why and how clinicians, researchers and national funding agencies could collectively reshape future obesity management strategies.

\section{STATUS QUO IS NOT AN OPTION}

In 1958, noted obesity researcher Dr Albert Stunkard stated, that 'Most obese persons will not stay in treatment for obesity. Of those who stay in treatment most will not lose weight and of those who do lose weight, most will regain it'. In 2012, 54-years and numerous studies later, we seem to be at the same point. For instance, consider a recent trial of weight loss and another of weight maintenance, which lasted an impressive 2 and 3 years, respectively. ${ }^{4,5}$ These trials conducted by some of the world's foremost experts, on highly-motivated individuals, who were closely monitored and supervised, resulted in about $4 \mathrm{~kg}$ reduction from about $100 \mathrm{~kg}$ starting body weight. While the results were statistically significant, their biological significance may be questioned. Will an approach that costs millions of dollars to produce less than $4 \%$ weight loss or maintenance, succeed in combating the global obesity epidemic in the free-living population, where the facilities, the expertise of health-care professionals, and the motivation of subjects is likely to be inferior to that in these studies?

For a wider reach, obesity management approaches that can be delivered effectively in a primary care setup are required. In fact, long-term weight loss in primary care practice was recently tested. ${ }^{6}$ After an intensive behavioral counseling, diet and drug treatment for 2 years, about $30 \%$ of the subjects lost $\geqslant 5 \%$ body weight. ${ }^{6}$ The authors concluded that 'primary care practices can initiate effective medical management of obesity'. Sadly, another interpretation of these results is that 'despite the intensive treatment, about $70 \%$ of the participants could not even achieve $5 \%$ of weight loss', -a routinely used criterion for success. Moreover, a recent study proposes that about $10-40 \%$ weight loss is needed for a long-term favorable effect on obesityrelated risk factors. ${ }^{7}$ Except for obesity surgery, such large weight losses are not routinely attainable, which perhaps leads to settling for $5 \%$ weight loss as success, but even that is not easily attainable.

Unfortunately, it appears intuitive to consider an obesogenic lifestyle as the root cause of obesity, and deceptively easy to modify. However, over 5 decades of manipulation of diet and activity, including composition, quantity and duration, has repeatedly failed to modify behavior in a biologically significant manner for the majority of people over an extended period of time. Albert Einstein is credited for the quote that 'insanity is doing the same thing over and over again and expecting a different result'. 


\section{THE ALTERNATIVES}

To be sure, despite their limitations, lifestyle modification and obesity drugs benefit some responders, and they are the best medical treatment options currently available to clinicians. Pharmacotherapy and obesity surgery are reasonably effective, but require a careful selection of recipients, which limits their wider application. A substantial shift in research focus is required to effectively combat global obesity in the future. A first step is to recognize the heterogeneity of obesity. Obesity, like cancer, or jaundice, has multiple contributors, and varied phenotypic and genotypic expressions. Individual weight loss or gain responses and even the metabolic benefits from exercise vary hugely and show an underlying genetic component. ${ }^{8,9}$ Obesity results from positive energy balance, but the factors that contribute to this energy surplus may vary. In addition to the 'usual suspects' of overeating and under-exercising, at least 10 non-conventional putative contributors of obesity are described in detail. ${ }^{10} \mathrm{~A}$ better understanding of such contributors to obesity should lead to cause-specific prevention or treatment strategies, which are likely to be more effective than the current non-specific blanket treatment of obesity.

Some putative contributors of obesity, such as 'assortative mating', ${ }^{10}$ could be hard to control. Whereas, some other putative contributors such as sleep debt, endocrine disrupters ${ }^{10}$ or certain infections, ${ }^{11}$ offer great examples for cause-specific prevention or treatment interventions. For instance, if a certain virus infection contributes to adiposity, a vaccine may effectively prevent and an antiviral agent may treat this subtype of obesity. Clearly, such an approach will be different than the conventional practice and yet may yield better results.

Even when the multi-factorial etiology of obesity is acknowledged, the role of genetic or biological contributors is frequently downplayed, in favor of obesogenic behavior, implied to be under complete volitional control. However, evidence indicates that food intake, or rewarding feelings after physical activity may be driven by upstream genetic or biological factors. ${ }^{12}$ For example, for many obese individuals, appetite and food intake regulating hormones such as Peptide YY, or ghrelin, function suboptimally. ${ }^{13,14}$ Hence, effective control of food intake may require targeting the abnormal responses of satiety hormones. Volitional modification of eating behavior is unlikely to succeed in such individuals.

An increase in adiposity in proportion to positive energy balance is understandable. Therefore, instead of focusing exclusively on those who gain weight in response to positive energy balance, another approach may be to study individuals who are relatively resistant to weight gain, as reported in an overfeeding study of twins. ${ }^{9}$ This may help to identify specific metabolic targets to attenuate weight gain.

In summary, it is hard to settle for conventional treatment approaches when non-responders or poor responders outnumber the responders. The assumption that the development of obesity or its reversal is only controlled by volitionally driven behavior has prompted intuitive-sounding conventional treatment approaches. These approaches have not had a meaningful impact on the global obesity epidemic. There are no easy answers to this complex issue, and success is not assured, but it is unlikely to come by trying incremental changes and variations of currently ineffective strategies. It is time to change course substantially by considering cause-specific prevention and treatment approaches. A first step is to identify hitherto unknown or under-recognized, yet modifiable factors that contribute to obesity by influencing behavior or, independent of it. National funding agencies such as the National Institutes of Health could encourage research in this area, by generating consensus, as initiated by a recent conference (http://calendar.nih.gov/app/MCallnfoView.aspx?evtID=20017) and providing directed funding. Clinicians could contribute by noting in their patients any unconventional factors or patterns that may seem to contribute to weight change. But, insightful input may result only if unconventional possibilities are entertained. In the field of obesity research and management, commonsense may not always make sense.

\section{ACKNOWLEDGEMENTS}

Dr Dhurandhar holds the following United States Patents: Patent number 6127113: Viral obesity methods and compositions. Patent number 6664050: Viral obesity methods and compositions. Patent number US 8008436B2, dated 30 August, 2011: Adenovirus 36 E4orf1 gene and protein and their uses. Provisional patent filed: Adenovirus Ad36E4orf1 protein for prevention and treatment of non-alcoholic fatty liver disease, July 2010. Provisional patent filed: Enhanced glycemic control using Ad36E4orf1 and AKT1 Inhibitor, January 2012.

NV Dhurandhar
Infection and Obesity, Pennington Biomedical Research Center,
Louisiana State University System, Baton Rouge, LA, USA
E-mail: nikhil.dhurandhar@PBRC.EDU

\section{REFERENCES}

1 Loeber S, Grosshans M, Korucuoglu O, Vollmert C, Vollstadt-Klein S, Schneider S et al. Impairment of inhibitory control in response to food-associated cues and attentional bias of obese participants and normal-weight controls. Int J Obes 2012; 36: 1334-1339.

2 Girz L, Polivy J, Herman CP, Lee $\mathrm{H}$. The effects of calorie information on food selection and intake. Int J Obes 2012; 36: 1340-1345.

3 Swartz JJ, Braxton D, Viera AJ. Calorie menu labeling on quick-service restaurant menus: an updated systematic review of the literature. Int J Behav Nutr Phys Act 2011; 8: 135.

4 Sacks FM, Bray GA, Carey VJ, Smith SR, Ryan DH, Anton SD et al. Comparison of weight-loss diets with different compositions of fat, protein, and carbohydrates. N Engl J Med 2009; 360: 859-873.

5 Svetkey LP, Stevens VJ, Brantley PJ, Appel LJ, Hollis JF, Loria CM et al. Comparison of strategies for sustaining weight loss: the weight loss maintenance randomized controlled trial. JAMA 2008; 299: 1139-1148.

6 Ryan DH, Johnson WD, Myers VH, Prather TL, McGlone MM, Rood J et al. Nonsurgical weight loss for extreme obesity in primary care settings: results of the Louisiana Obese Subjects Study. Arch Intern Med 2010; 170: 146-154.

7 Sjostrom CD, Lystig T, Lindroos AK. Impact of weight change, secular trends and ageing on cardiovascular risk factors: 10-year experiences from the SOS study. Int J Obes (Lond) 2011; 35: 1413-1420.

8 Bouchard C, Sarzynski MA, Rice TK, Kraus WE, Church TS, Sung YJ et al. Genomic predictors of the maximal $\mathrm{O}$ uptake response to standardized exercise training programs. J Appl Physiol 2011; 110: 1160-1170.

9 Bouchard C, Tremblay A, Despres JP, Nadeau A, Lupien PJ, Theriault G et al. The response to long-term overfeeding in identical twins. N Engl J Med 1990; 322: 1477-1482.

10 McAllister EJ, Dhurandhar NV, Keith SW, Aronne LJ, Barger J, Baskin M et al. Ten putative contributors to the obesity epidemic. Crit Rev Food Sci Nutr 2009; 49: 868-913.

11 Dhurandhar NV. A framework for identification of infections that contribute to human obesity. Lancet Infect Dis 2011; 11: 963-969.

$12 \mathrm{Knab}$ AM, Lightfoot JT. Does the difference between physically active and couch potato lie in the dopamine system? Int J Biol Sci 2010; 6: 133-150.

13 Carroll JF, Kaiser KA, Franks SF, Deere C, Caffrey JL. Influence of BMI and gender on postprandial hormone responses. Obesity 2007; 15: 2974-2983.

14 le Roux CW, Batterham RL, Aylwin SJ, Patterson M, Borg CM, Wynne KJ et al. Attenuated peptide $Y Y$ release in obese subjects is associated with reduced satiety. Endocrinology 2006; 147: 3-8. 